\title{
Linx
}

Revue des linguistes de l'université Paris X Nanterre

70-71| 2014

Variations sémantiques et syntaxiques : aspects d'une théorie de l'invariance

\section{Une réussite sans égal : analyse compositionnelle du russe UDAT'SJA « réussir »}

\section{Rémi Camus}

\section{(2) OpenEdition}

\section{Journals}

Édition électronique

URL : http://journals.openedition.org/linx/1572

DOI : $10.4000 /$ linx.1572

ISSN : 2118-9692

Éditeur

Presses universitaires de Paris Nanterre

Édition imprimée

Date de publication : 1 septembre 2014

Pagination : 107-124

ISSN : 0246-8743

Référence électronique

Rémi Camus, "Une réussite sans égal : analyse compositionnelle du russe UDAT'SJA « réussir » », Linx [En ligne], 70-71 | 2014, mis en ligne le 01 mars 2015, consulté le 23 avril 2019. URL : http:// journals.openedition.org/linx/1572; DOI : 10.4000/linx.1572 


\title{
Une réussite sans égal : analyse compositionnelle du russe $U D A T ' S J A$ " réussir »
}

\author{
Rémi Camus, Inalco \\ MoDyCo CNRS, Université Paris Ouest Nanterre la Défense
}

\section{Les termes du problème}

Il y a réussir et réussir : réussir son examen de passage peut s'effectuer sans panache, avec la note minimale acceptable; c'est en quelque sorte s'en acquitter (en anglais : to pass an exam). En revanche, réussir sa tarte impose l'excellence : une tarte réussie est un bonne tarte.

Le russe utilise dans les deux cas des composés préverbés du verbe de base dat' «donner, laisser, faire ». La tournure russe est transitive pour «j'ai passé mon examen » (ja sdal / sdaval ekzamen), et le verbe présente le préfixe $s$ - marquant un accomplissement ; il faut en revanche une tournure intransitive pour "J'ai réussi ma tarte», sous une forme qu'on peut rendre littéralement «La tarte s'est donnée à moi » (Tort mne udalsja). Avec la structure transitive, le verbe accepte aussi bien un verbe dit "perfectif » (sdal) qu'un verbe «imperfectif » (le dérivé sdaval qui, s'agissant d'un examen ponctuel, signifie simplement qu'on s'y est présenté); la seconde nécessite un verbe perfectif (sauf contexte itératif : j'avais coutume de réussir mes tartes). C'est cette seconde tournure qui justifie la présente enquête : en apparence limpide, elle devient problématique une fois réinscrite dans le système grammatical russe. 
Le verbe $u d a t$ 'sja « être une réussite » est constitué de trois éléments :

1) La particule d'origine pronominale $-s j a($ variante $:-s$ ) qui est toujours postfixée au mot verbal ;

2) Le morphème $u$ fonctionnant tantôt comme préposition, tantôt comme préfixe, surtout verbal, par exemple :

(a) Préposition :
u kamin-a
u cheminée-GEN.SING
« auprès de la cheminée »

(b) Préposition :

$\begin{array}{lll}\text { Knig-a } & u & \text { men-ja } \\ \text { livre-NOM.SING } & u & \text { moi-GEN }\end{array}$

«Le livre est chez moi » ou «C'est moi qui ai le livre»

(c) Préfixe :

- verbal : letet' « voler » => u-letet' « s'envoler »;

- nominal : radical de letet' => u-lèt! «C'est extra!»

3) Le verbe dat' ' donner, laisser, faire »

Or, malgré l'existence de verbes en apparence dérivés avec les mêmes éléments $u$ - et -sja, on montre aisément qu'il n'existe pas en russe de formations similaires au verbe udat'sja. Les particularités de udat'sja apparaissent bien en comparaison avec les mots construits à partir de nesti "porter, apporter, supporter, etc. ». On a la combinatoire lacunaire suivante (traductions ci-après) :

$$
\begin{array}{lll}
\text { (1) nesti / nesti-s' } & \text { dat' } \\
\text { u-nesti / u-nesti-s, } & { }^{\prime} \text { udat }, ~ / ~ & \text { dat'-sja } \\
\text { u-dat'-sja }
\end{array}
$$

Nesti est compatible avec toute la combinatoire (les formes pronominales signifiant un déplacement du sujet : nestis" "filer», unestis" " filer, disparaître»); au simplex dat' répond dat'sja utilisé dans des tours passifs. En revanche l'intransitif udat'sja « réussir » n'a pas de correspondant sans postfixe en russe moderne : *udat' est mal construit, ne fournit pas d'analogue syntaxique au français réussir [quelque chose] (transitif). Quant aux autres verbes composés obtenus par ajout concomitant de $u$ - et de -sja, leur radical verbal n'est pas comparable à dat': ils sont intransitifs y compris sous leur forme non composée. Par exemple : leč" « s'allonger » => uleč'sja «prendre ses aises » ou « retomber (des passions)».

La progression de l'analyse est imposée par le problème lui-même : il faut tout d'abord circonscrire le fonctionnement de chacune des unités en jeu (chapitres 2. à 4. ci-après), puis en venir à leur combinatoire (chapitre 5. ${ }^{1}$. 


\section{Dat'2}

On doit à Apresjan (par exemple Apresjan, 1995) une glose calquant celle des analyses componentielles de l'anglais give: "Faire en sorte qu'autrui ait/possède ». Cette définition est parfois interprétée comme une double relation :

- relation de causation; Apresjan lui-même utilise une autre formulation requérant une version russisée de l'opérateur CAUSE, malaisée à traduire :

« Kauzirovat' X imet' A i kauzirovat' sebja ne imet' A » (Apresjan, $1995: 304)$

«Causer X posséder A \& Causer soi-même ne pas posséder A. »

- relation de possession d'autre part. Dat' est rangé parmi les «verbes de possession » (glagoly obladanija) par de nombreux auteurs (cf. R. Rozina, 2005).

La critique de l'analyse en «causer » et " posséder » nous fournira un accès au fonctionnement invariant de dat'.

\subsection{Dat' excède la sphère de la possession}

Admettons provisoirement que la notion de possession soit utile pour décrire les emplois de dat' marquant une transmission, quitte à assouplir la formule ci-dessus : dat' n'implique pas nécessairement qu'on se déprenne de ce que l'on donne : dat' sovet « donner un conseil », dat' ruku « donner la main / le bras » etc.

Elle n'en est pas moins inapplicable dans de très nombreux autres emplois qui ne signifient nullement que quoi que ce soit transite d'un sujet à un autre. Dans les exemples suivants, aucun destinataire n'est ni exprimé, ni même restituable par l'interprétation :

(2) Корабль дал течь, но мы на плаву.

Korabl-' da-1 teč, no my na plavu.

Navire dat'-PASS fuite, mais nous à flot.

«Le navire prend l'eau (a une voie d'eau), mais nous restons à flot »

(3) Он дал последний рывок и вырвался вперед.

On da-1 poslednij ryvok ivyrvalsja vperëd.

Il dat'-PASS dernier à-coup et s'échappa en-avant

« Il donna un dernier coup de reins et se lança dans une échappée. »

(4) Ну ты дал!

$\mathrm{Nu}$ ty dal!

Alors tu dat'-PASS

«Alors là, tu as fait fort!»

que minimalement justifié. Quelques références bibliographiques en notes infrapaginales visent à compenser les inévitables raccourcis.

2 Cf. Camus 2007, 2008 
(2) signifie l'entrée du sujet korabl" "bateau » dans un état qui serait glosé par la construction de localisation (2') :

$$
\begin{array}{ll}
\text { (2') } & V \quad \text { korable te } \breve{C} . \\
& \text { Dans bateau-PREP fuite. } \\
& \text { «Le bateau a une voie d'eau. » }
\end{array}
$$

(3) prédique un événement (une "occurrence» de procès) : le radical du substantif suffixé ryv-ok "un à-coup» se retrouve dans le verbe rvat'sja "s'élancer", vyrvat'sja «s'échapper, s'extraire ».

Enfin, d'une ou plusieurs actions qu'on n'explicite pas, l'exclamation (4) ne relève que la part déterminante prise par son agent («tu»), source d'admiration, de stupéfaction ou encore de réprobation.

Qualifier ces emplois de collocations (2,3) ou d'expression exclamative (4) laisse entière la question du rôle accordé à dat'.

\subsection{Dat' n'est pas " cause to have"}

La causation n'est pas inscrite dans la glose russe procurée par Apresjan: «Faire en sorte qu'autrui possède ». La locution « afin que, en sorte que + subj.» (en russe : take čtoby) peut être prise au sens strict: la possession devient possible grâce à dat', mais elle n'est aucunement construite par dat'.

Par exemple : Mne dali 20 kop. «on m’a donné 20 kopecks » n'implique pas nécessairement «j’ai 20 kopecks». Un contraste fréquent dans les textes consiste précisément à opposer cette séquence à sa converse Ja vrjal 20 kop. «j’ai pris 20 kopecks » qui fait de ja «je » un détenteur-possesseur de 20 kopecks. Dans l'exemple suivant, les pièces données sont tout simplement jetées ostensiblement dans l'assiette :

(5) В буфете с 20 коп. дали 30 коп. сдачи, но я не взял и мне за это выдали старый и грязный голландский сыр. ${ }^{3}$

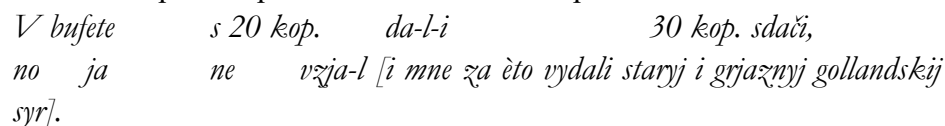

Au buffet de 20 kop. dat'-PASS-PLUR 30 kop. de monnaie, mais je NEG prendre-PASS [...]

(Époque soviétique) Au buffet on m'a rendu 30 kopecks sur 20, mais je ne les ai pas pris [, ce qui m'a valu un morceau de gouda vieux et sale].

Si le verbe dat' suffisait en soi à transformer le destinataire en détenteur ou possesseur, on ne voit pas comment celui-ci aurait la latitude de refuser de devenir ce détenteur ou possesseur; le verbe et son contexte seraient en totale contradiction.

\footnotetext{
3 Tous les exemples ont été validés par plusieurs informateurs. Sauf indication du contraire, ils proviennent de textes en ligne.
} 
Plus généralement, la thèse qui interprète dat' sur le modèle de la transmission réussie ("udav̌sajasja peredača », Rozina, 2005) revient à attribuer à la sémantique du verbe tous les ingrédients d'un seul des scénarios qu'il contribue à décrire. Dans l'optique d'une lecture compositionnelle de udat'sja, la définition doit ne retenir des emplois de dat' que ce qui relève en propre de ce lexème. Dat' n'exprime pas plus en soi un acte de transmission ou même d'acquisition qu'il n'exprimait l'apparition d'une fuite d'eau, un à-coup ou quelque action d'éclat. S'agissant de ces procès notés désormais $\mathbf{P}$, chacun possédant ses propres modalités de validation, nous dirons que dat' introduit un terme $\mathbf{D}$ qui détermine la validation de $\mathbf{P}$ (au sens « $P$ est le cas").

D peut s'interpréter comme déclencheur ou actualisateur suivant qu'il est requis contextuellement ou non, qu'il possède a priori un rôle d' «impedimentum» (facteur d'empêchement) potentiel, ou que ce rôle ne soit envisageable qu'après coup (« si $\mathbf{D}$ n'avait pas été là... »). Les interprétations des emplois auxiliés de dat' donneront une idée de cette variation ; soit la séquence suivante :

(6) Дай знать о себе!

$$
\begin{array}{llll}
\text { Daj } & \text { znat' } & \text { sebe. } \\
\text { dat'IMP } & \text { savoir/connaître } & \text { au_sujet_de } & \text { soi. }
\end{array}
$$

Cette séquence peut correspondre à au moins trois types de relation entre l'interlocuteur (correspondant à $\mathbf{D}$ ) et le locuteur, et trois traductions (entre crochets sont ajoutées des indications sur les contextes):

- Injonction / incitation - l'interlocuteur est absent, on lui demande d'apparaitre. «Manifeste-toi [si tu es là]. »

cf. Daj posmotret"! littéralement Donne regarder qui peut se rendre «Fais voir!», ou «Montre!». Aucune résistance de $\mathbf{D}$ n'est a priori envisagée.

- Mettre sur la bonne voie - l'interlocuteur, bel et bien présent, est toutefois dans une position d'indétermination quant au procès ; il est enjoint à faire preuve d'initiative :

«Fais toi connaître (c'est dans ton intérêt)!»

Cette valeur se retrouve dans Dajte ja Vas provožu! littéralement Donnez je vous raccompagnerai, qui correspond au français «Allez, je vous raccompagne », ou même à «Laissez-moi vous raccompagner».

- Prévenir un refus - on prie l'interlocuteur de ne pas s'opposer au procès :

«Donne de tes nouvelles [je t'en prie]!»

cf. Daj vyskazat'sja! littéralement Donne m'exprimer « Laisse-moi m'exprimer », c'est-àdire « Ne m'empêche pas de parler!».

Ces emplois modaux illustrent en outre le point suivant : c'est fondamentalement une relation binaire entre le terme $\mathbf{D}$ et une relation prédicative $\mathbf{P}$ non actualisée qui est mise en jeu par dat'. L'analyse binaire est également licite pour l'exemple emblématique de la valeur de transmission : 
(7) Саша дал Пете книгу.

\begin{tabular}{|c|c|c|c|}
\hline $\operatorname{Saŕa}(=\mathbf{D})$ & dal & $\underline{\text { Pet-e }}$ & $\underline{\text { knig-u }}$ \\
\hline Sacha & dat'-PASS & Pierre-DAT & livre-ACC \\
\hline
\end{tabular}

Les termes soulignés constituent un état de choses $« \mathbf{P} »$ complexe : $\mathbf{P}$ correspond à la mise en relation du destinataire représenté par un syntagme nominal au datif, et de l'objet cédé qui est représenté par un composant au cas accusatif ; D s'interpose :

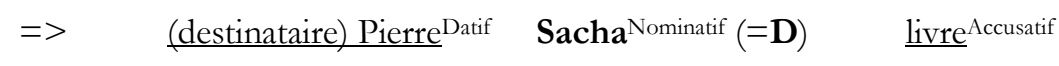

Ainsi, l'apport de dat' réside dans l'introduction de Sacha sur qui, désormais, repose la relation livre/Pierre. Des exemples tels que (5) montrent clairement que l'introduction de $\mathbf{D}$ par DAT' ne suffit pas en soi à transformer Pierre, du destinataire qu'il était, en possesseur ou détenteur. Autrement dit, le contexte peut confirmer ou infirmer la transformation du destinataire (marqué par le datif) en possesseur, puisque dat' n'affecte celui-ci qu'indirectement; dat' a pour fonction d'introduire une instance extérieure à $\mathbf{P}$, et cette instance tierce devient l'unique source de validation de $\mathbf{P}$. Un don a bien lieu, que Pierre entre en possession du livre ou non. Où le don confine à l'aliénation...

Ajoutons un argument fort en faveur de l'hypothèse avancée : dans les emplois du verbe dat' à des formes finies, $\mathbf{D}$ est toujours le sujet syntaxique. Autrement dit, le russe ignore les emplois impersonnels de dat' (on laisse ici de côté des attestations dialectales d'emplois impersonnels de dat', rares et circonscrites).

Les limites du présent article ne permettent pas de passer en revue un échantillon représentatif des emplois du verbe dat' donné comme un des 100 vocables les plus fréquents du russe contemporain (Zasorina, 1977). En revanche, l'hypothèse peut être illustrée à petite échelle grâce à des manipulations élémentaires sur un sous-corpus :

$$
\begin{array}{ll}
\text { (2, déjà cité) Корабль дал течь, но мы на плаву } & \\
& \text { Korabl da-l tec, no my na plavu. } \\
& \text { Navire dat'-PASS fuite, mais nous à flot } \\
& \text { «Le navire prend l'eau (a une voie d'eau), mais nous restons à flot » }
\end{array}
$$

Korabl' « navire» peut être remplacé mutatis mutandis par bak « réservoir» ou akvarium «aquarium»: "le réservoir / l'aquarium a une fuite»; comparer avec l'impossibilité de (8) :

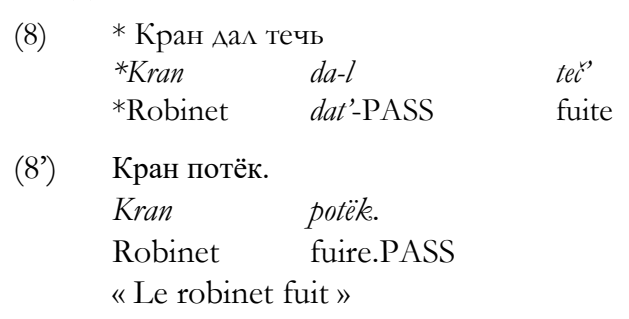

«Bateau », «aquarium» et « réservoir» se définissent par leur résistance au passage de l'eau : c'est à ce titre que leur prise en compte est déterminante pour la fuite 
(et il ne viendrait nullement à l'esprit de les décrire comme causateurs). Il n'en va pas de même du robinet, mécanisme qui stoppe ou ne stoppe pas l'eau suivant l'état dans lequel on le place, d'où l'impossibilité signalée en (8).

(9) Я я дй дал пощёчину.

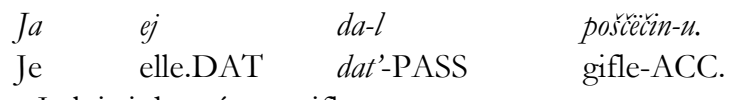

« Je lui ai donné une gifle. »

(9’) ??Я ей дал поцелуй. ${ }^{4}$

??Ja ej da-l poceluj.

??Je elle.DAT dat'-PASS baiser.

Cette séquence n'est guère acceptable, sauf réinterprétation contextuelle de poceluj au sens de «cadeau », cf. podarit' poceluj « offrir un baiser », " donner l'offrande d'un baiser », ou encore en présence de déterminants qualifiant poceluj: dat' poceluj Iudy «donner le baiser de Judas ». Alors qu'en français, Je lui ai donné un baiser ne pose pas plus de difficulté que ses traductions littérales dans de nombreuses langues ${ }^{5}$.

Le baiser, même donné à la dérobade ou déposé furtivement, est un événement (cf. le préfixe $p o-$ dans $p o c e l y^{6}$ ) qui implique la participation ou le bon vouloir du destinataire (un poceluj se fait à deux). Alors qu'avec dat', la relation est a priori contingente, la cible doit pouvoir se soustraire à moins qu'il ne s'agisse d'un baiser qu'on vole (ukeradennyj poceluj), et ni le français donner, ni a fortiori le russe dat' ne s'emploient. On vérifie de même :

(10) пнуть кого-нибудь ногой

pnut' kogo-nibud' nog-oj

botter quelqu'un.ACC pied-INSTR

«botter quelqu'un au train »

$\left(10^{\prime}\right)$ дать кому-нибудь пинка

dat' komu-nibud' pink-a

dat' quelqu'un.DAT coup-de-pied-GEN

« donner un coup de pied à quelqu'un »

(11) пнуть ногой в дверь

pnut' nogoj $v$ dver'

botter pied-INSTR dans porte

« envoyer un coup de pied dans la porte »

$4 \quad$ Contrainte relevée par O. N. Selivërstova $(1975,2004)$.

All. ein Küsschen geben, angl. to give a kiss, persan bus (anciennement : qubla) dädan, esp. dar un beso; hors domaine indoeuropéen : chin. 我给了她一个吻 Wǒ gĕi le tā yĩ ge wèn lit. je à/donner lui particule un spécificateur baiser, hébr. לתת נש יקה latet nešikah «donner [un] baiser» etc. On n'est donc pas étonné de trouver épisodiquement des occurrences de l'expression calquée en russe dans des traductions.

6 Sur la dimension événementielle de po-, cf. Camus (1998). 


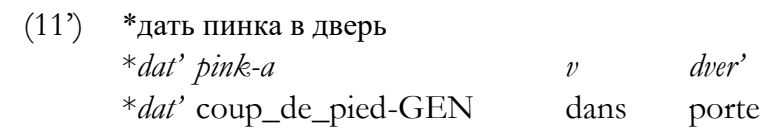

L'impossibilité de (11') avec une cible inanimée (dver' " [la] porte ») confirme que avec le verbe dat', mais non avec pnut' « botter », la cible de l'action est en mesure d'esquiver le coup. Au sens d'« asséner (un coup) », dat' confère au sujet l'entière maitrise de l'action.

Enfin, pour montrer sur un seul exemple comment se met en œuvre l'hypothèse que nous construisons dans ces lignes lorsqu'il s'agit d'emplois non spatiaux :

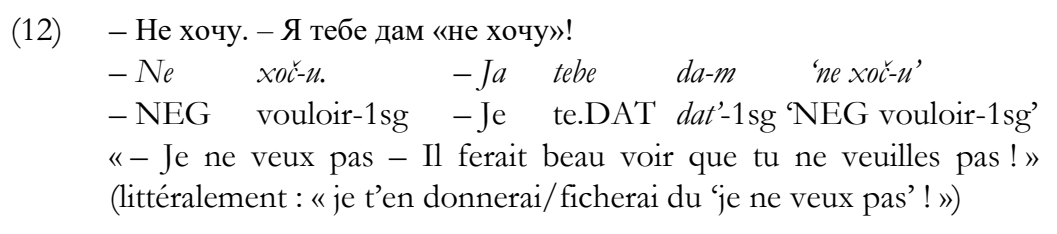

Il s'agit d'un exemple parmi d'autres de la valeur antiphrastique des verbes perfectifs conjugués : tebe dam, litt. "je te donnerai», est ironique. Face à une position jugée exorbitante (ne хос̆ « je ne veux pas » dont la reprise est obligatoire), $\mathbf{D}$ réaffirme son droit souverain d'accepter ou pas les caprices d'autrui : un refus n'est licite que s'il émane de $\mathbf{D}$; cela revient par conséquent à une fin de non-recevoir.

Nous avons jusqu'à présent envisagé le contrecoup de dat' sur la représentation d'un procès $\mathbf{P}$ : dat' introduit une terme $\mathbf{D}$ extérieur à $\mathbf{P}$ qui détermine sa validation. L'ajout du préfixe $u$-ajoute à son tour, nous allons le voir, un autre terme ; mais celuici est étranger à dat'.

\section{Le préfixe $u^{-7}$}

Tout en associant $u$ - à la notion de résultat, la littérature ${ }^{8}$ souligne le caractère souvent imprévisible et paradoxal des valeurs engendrées par $u$ - en fonction des bases verbales et des constructions. Un état préjudiciable dans učinit" "faire (quelque chose de mauvais)» construit sur čnit' "réparer», mais souhaitable dans usadit' "asseoir (confortablement) » répondant à sadit'sja « s'asseoir»; réduction dans uvarit' lorsqu'il signifie «faire réduire en portant à ébullition (varit')» alors que ce même $u$ - figure comme préverbe dans uvelicit' "agrandir» (cf. adjectif velikij « grand»); éloignement dans uletet' "s'envoler » sur letet' "voler », mais persistance dans usidet' " rester assis » sur sidet' " être assis », etc.

Je reprendrai ici, puis illustrerai brièvement l'hypothèse avancée dans l'ouvrage Dobrušina, Mellina, Paillard (2001). Cette hypothèse repose sur la notion d'efficacité : en comparant govorit' " parler, dire » et ugovorit' " convaincre, persuader », les auteurs

\footnotetext{
$7 \quad$ Cette section utilise les descriptions procurées par K. Rey (sans date), E. R. Dobrušina, E. Melina, D. Paillard (2001). On se reportera à ce dernier ouvrage pour une critique argumentée des approches alternatives Gorelik (2001) et Zaliznjak (2001).

$8 \quad$ cf. la synthèse procurée par Anna A. Zaliznjak (2001).
} 
relèvent que la conviction ne peut pas s'obtenir à partir de l'activité du " parleur » ellemême; l'efficacité de la parole dépend de l'interlocuteur qui, seul, peut signifier par son acquiescement éventuel qu'il est convaincu.

Les auteurs reprennent cette observation locale comme point de départ à leur analyse de $u$. Ils l'adaptent au schéma relationnel proposé par D. Paillard pour rendre compte des des préverbes du russe ; suivant ce schéma, tout préverbe se représente comme un relateur R portant sur deux termes. Dans ce cadre, $u$ - est constitutif de la relation «Base verbale » $\mathbf{R}$ « $\mathrm{Y} »$ :

- Le résultat $\mathbf{Y}$ correspond à l'état actuel d'un élément donné ;

- Y n'est pas le développement attendu du procès désigné par la base verbale ;

- C'est à partir de $\mathbf{Y}$ que se détermine la quantité de procès mise en jeu.

Il s'ensuit que la construction de $\mathbf{Y}$ mobilise un point de vue autre que celui donné par les ingrédients de la base verbale.

\section{4. *udat'vs udat'sja}

L'impossibilité de *udat' découle de la combinaison des deux caractérisations données. Il y a en effet contradiction entre :

- D est condition sine qua non de la réalisation de $\mathbf{P}(=$ dat' $)$;

- la mesure de la réalisation de $\mathbf{P}$ est extérieure aux éléments donnés par la base verbale $\operatorname{dat}^{\prime}(=u-)$.

En d'autres termes, l'efficacité $(u-)$ implique un résultat dissocié des efforts déployés par l'agent du procès, alors que dat' marque que c'est ce même agent qui détermine la validation de l'état de choses $\mathbf{P}$.

\section{5. $-s j a /-s^{\prime}$}

Rappelons la distribution lacunaire: *udat' mais udat'sja "être une réussite », unesti « emporter » et unestis' " partir ». Autrement dit, dans le cas de dat', la présence du postfixe pronominal $-s j a /-s$ ' rend possible l'ajout du préverbe $u$.

Une des propriétés principales du postfixe - sja/-s' est de rendre un verbe intransitif. Le verbe dat' impliquant une relation entre deux termes (dont un de nature prédicative : $\mathbf{P}$ ), cela signifie en l'occurrence ceci : quelle que soit la voix - active, passive ou moyenne -, -sja/-s' marque que l'un de ces deux termes est a priori affecté à une position, sujet ou complément, mais n'y est pas exprimé ; il n'en est pas moins présent dans l'interprétation, parfois récupérable au sein de l'énoncé9.

9 Sur ce point également, je reprends la présentation donnée dans l'ouvrage Dobrušina, Mellina, Paillard (2001) qui parle de « vidage » (otstranenie) desdites places. Sont laissés de côté certains problèmes non pertinents pour notre enjeu : quid des verbes pronominaux bojat'sja « avoir peur de » et slušat'sja "obéir » qui sont transitifs avec les compléments animés? quid des verbes en -sja sans correspondant non postfixé ? etc. 


\section{1. dat'sja}

- dat'sja interdit toute interprétation réfléchie qui ferait coïncider le procès $\mathbf{P}$ avec le terme $\mathbf{D}$ puisque ce verbe, précisément, a pour fonction de conserver un écart entre $\mathbf{P}$ et le terme $\mathbf{D}$ qui détermine sa validation.

Dès lors, dans tous les cas, la combinaison de -sja/-s' avec dat' signifie que c'est le terme $\mathbf{D}$ déterminant $\mathbf{P}$ qui n'occupe pas la position qui lui est réservée : ce cas de figure recoupe plusieurs variétés de voix passive. Nous distinguerons un passif qui promeut le second terme, et un passif modal ${ }^{10}$.

\subsubsection{Passif promouvant le second terme}

- L'absence de syntagme nominal représentant $\mathbf{D}$ peut déboucher sur une interprétation générique de $\mathbf{D}$ : quiconque est - ou a vocation à être - agent du procès. Par exemple :

(13) Ему дастся определенное время на подготовку.

$\begin{array}{llllll}\text { Emu } & \text { da-st-sja } & \text { opredelënnoe vremja na podgotovku } & \text { na } \\ \text { lui.DAT } & \text { dat'-3sg-SJA } & \text { certain } & \text { temps pour préparation. }\end{array}$

Contexte :

«Voici quelques-unes des épreuves proposées aux impétrants. (...) Le candidat a pour consigne d'effectuer sa présentation devant un jury. Il lui sera donné un temps de préparation. Pendant sa présentation, il peut utiliser divers moyens, en particulier un projecteur. »

En vertu d'une contrainte générale portant sur la catégorie de l'aspect verbal en russe, cet emploi passif du verbe perfectif dat'sja est incompatible avec le passé ; on trouvera donc exclusivement les formes dites « de présent »: « présent-futur » de dat'sja (d'où la traduction de (13) au moyen du futur simple français), ou encore « présent» de son dérivé imperfectif davat'sja ${ }^{11}$ comme dans (14) :

10 Dat'sja ne possède pas d'emplois réfléchis : $\mathbf{D}$ est par définition extérieur au procès $\mathbf{P}$ qu'il conditionne et ne peut coïncider avec lui. En revanche, une interprétation réfléchie est possible avec le pronom objet sebja. J'en cite in extenso une rare illustration (à l'imperfectif : davat' sebja, lit. "donner soi[-même] »), description de l'Eucharistie - don ou partage bénévole de soi :

В Иисусе Христе, Воплощенной любви Бога, "eros-agape" принимает наиболее радикальную форму. В смерти на кресте Иисус, жертвуя Себя ради спасения человечества, выразил любовь в наиболее возвышенной форме. Он гарантировал постоянное присутствие этого акта любви в Евхаристии, в которой под видами хлеба и вина Он дает Себя нам как манну, объединяющую нас с Ним. Участвуя в Евхаристии, мы также становимся вовлеченными в Его акт самопожертвования. Мы объединяемся с Ним и с теми, кому Он дает Себя. (Митрополит Тадеуш Кондрусевич, Краткий комментарий к энциклике Бенедикта XVI «Deus caritas est »)

«Dans Jésus-Christ, Amour de Dieu incarné, l' 'eros-agapê revêt sa forme la plus radicale. En mourant sur la croix, Jésus, par Son sacrifice pour l'humanité, a exprimé l'amour dans sa forme la plus haute. Il a garanti la présence ininterrompue de cet acte d'amour dans l'Eucharistie durant laquelle, sous les espèces du pain et du vin, il Se donne à nous comme une manne qui nous réunit avec lui. En prenant part à l'Eucharistie, nous participons à notre tour à Son sacrifice. Nous faison un avec Lui et ceux auxquels Il Se donne. »

$11 \quad$ Voici un contre-exemple apparent :

tous. »)

[titre d'article] Attestacija dalas' ne vsem (litt. «L'attestation/accréditation se-donna NEG à- 
Une réussite sans égal : analyse compositionnelle du russe UDAT'SJA « réussir »

(14) (Деньги - это вечный двигатель теневой экономики.) Именно ими даются взятки (и оплачиваются наркотики).

(Den'gi - èto večnyj dvigatel' tenevoj èkonomiki.)

Imenno imi dajutsja vajatki

(i oplačivajutsja narkotiki)

Nommément eux.INSTR davat'-3.PLUR-SJA pots-de-vin (...) «(L'argent est le moteur perpétuel de l'économie parallèle).

C'est par son truchement que se versent (lit. «se-donnent») les potsde-vin et que se règlent les stupéfiants. »

- La réintroduction de $\mathbf{D}$ dans un « complément d'agent» à l'instrumental est caractéristique du registre didactique. On remarque le pluriel d'auteur dans (42) :

(15) В прямых скобках нами даются слова, необходимые для понимания текста.

Vprjamyx skobkax [] nami dajutsja slova, neobxodimye dlja ponimanija teksta.

En parenthèses carrées nous.INST davat'-3.PLUR-SJA mots, indispensables pour la compréhension du texte

«Entre parenthèses carrées nous donnons (lit. "par-nous se-donnent») les mots indispensables à la compréhension du texte. »

ou encore :

(16) Прокурор выясняет и дает оценку: кем из руководителей органа проверялись дела оперативного учета; какие указания ими давались в рамках оперативной разработки.

Prokuror vyjasnjaet i daet ocenku: kem iz rukovoditelej organa proverjalis' dela operativnogo ucëta; kakie ukazanija imi davalis' v ramkax operativnoj razrabotki.

«Le procureur détermine et qualifie : qui des responsables du service étaient en charge du contrôle des activités du service, quelles consignes furent délivrées par leurs soins (lit. quelles consignes par-eux sedonnaient) lors de la préparation des opérations. »

Il s'agit d'un jeu de mots qui projette davat' attestaciju «donner une attestation, accréditer » sur la valeur modale du passé du perfectif dat'sja impliquant des obstacles à surmonter (cf. infra), ce que confirme l'article:

Завершена аттестация руководителей больниц. Из 962 главврачей, подвергнутых «экзамену» Минздравом, только 90 процентов успешно с ним справились.

А вот 67 человек были отстранены от занимаемой должности в силу недостаточной профессиональной подготовки, отсутствия у них организаторских качеств или иных серьезных недостатков....

«La procédure d'accréditation des directeurs d'hôpitaux est close. Des 962 spécialistes soumis à 'l'examen' par le Ministère de la Santé, seulement $90 \%$ l'ont passé honorablement. Alors que les 67 restants ont été démis de leur fonctions pour cause de préparation professionnelle insuffisante, du manque de savoir-faire organisationnel ou autres défauts graves. » 


\subsubsection{Passif modal}

Dans cette rubrique, un facteur dirimant empêche $\mathbf{D}$ de remplir son rôle déterminant pour la réalisation de $\mathbf{P}$. Le référent de $\mathbf{D}$ s'identifie alors comme simple participant du procès $\mathbf{P}$, en bute à une résistance.

\section{- Procès impossible ou difficile}

Les énoncés de ce type mettent en scène une résistance non surmontée (énoncés négatifs), ou encore surmontée plus ou moins difficilement par le terme exprimé au datif : ils contiennent alors obligatoirement des compléments adverbiaux comme trudno "difficilement", dorogo "chèrement», kakoj cenoj «à quel prix» et même legko «facilement» dans la mesure où il implique une difficulté attendue. La nature du sujet syntaxique n'est pas fixée; il peut s'agir d'un agent potentiel ou d'un terme non agentif :

L'exemple (17) illustre, en contexte d'antagonisme, le cas où le sujet syntaxique renvoie à un animé ou assimilable :

(17) [Titre de brève] 19.11 20:44 - Самара не Аалась Аэрофмоту, отАав предпочтение KrasAir ("Ђ")

$$
\begin{array}{ll}
\begin{array}{l}
\text { Samara ne dalas' } \\
\text { otdav predpoćtenie KrasAir }
\end{array} & \text { Aèroflot-u, } \\
\text { Samara NEG dat'-PASS-FEM-SJA } & \text { Aèroflot-DAT } \\
\text { (...) } & \\
\text { "La compagnie Samara n'est pas passée sous le contrôle d'Aèroflot } \\
\text { et a préféré KrasAir. " }
\end{array}
$$

Contexte (Début de la brève) :

Как стало известно 'Ђ', менеджмент авиакомпании 'Самара' подписал договор о вхождении в альянс авиаперевозчиков, создаваемый компанией KrasAir. Подписание этого договора означает, что руководство 'Самары' нарушило соглашение с компанией 'Аэрофлот'

« Notre rédaction apprend que la direction de la compagnie d'aviation Samara a signé un accord la faisant entrer dans l'alliance de transporteurs aériens que crée la compagnie KrasAir (...) La signature de ce contrat signifie de la part de la direction de Samara la rupture de l'accord conclu avec la compagnie Aéroflot. »

Le sujet de dat'sja dans (17) n'est pas identifiable au constituant $\mathbf{D}$ qui détermine la validation du procès : étant donné le procès $\mathbf{P}$ décrivant la prise de contrôle de la firme par la compagnie aérienne Aèroflot, l'impossibilité d'assigner un élément de $\mathbf{P}$ au constituant $\mathbf{D}$ introduit par dat' appelle la négation : $\mathbf{P}$ n'a pas été validé. Comparer (17)-(18) à (19) qui contient le préverbé otd'atsja qu'on peut traduire effectivement «se donner à, se livrer à » avec une interprétation réfléchie du postfixe pronominal :

(18) Россия не Аалась Наполеону.

$$
\text { Rossija ne da-l-a-s' Napoleon-u }
$$


(19) Россия не отдалась в хищные мапы Запада

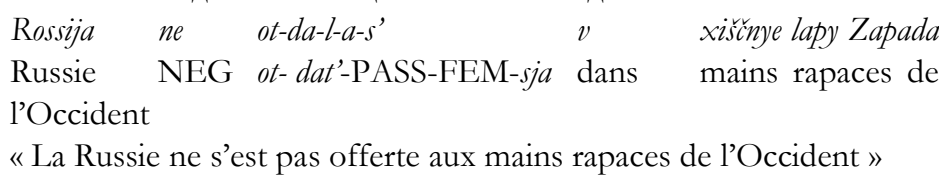

(18) constate que les efforts de Napoléon sont restés vains. C'est cet échec que rend la traduction «a su résister» qui s'impose hors contexte; toutefois, cette traduction attribue trop de responsabilité à la Russie : ne dalas' se borne à constater que Napoléon lui-même n'a pas été à la hauteur de ses ambitions. Il en va tout autrement dans (19) qui redonne l'initiative au sujet syntaxique «La Russie»: celle-ci est alors bien en position de succomber (Rossija otdalas' Zapadu «La Russie s'est offerte à l'Occident») ou résister.

Les exemples suivants décrivent des objectifs (difficiles) à atteindre ; les sujets syntaxiques représentent des inanimés. Dans ces emplois, le sujet peut être un terme exprimant en soi ou contextuellement un objectif: un obstacle à franchir ou à " prendre », une victoire, un record, un rêve etc.

(20) Индекс РТС прибавил около 3\% и превысил отметку 600 пунктов. Важный психологический барьер дался ему с трудом.

Indeks RTS pribavil okolo 3\% i prevysil otmetku 600 punktov.

Važnyj psixologičskij bar'er dalsja emu s trudom.

Importante barrière psychologique dat-PASS-sja lui.DAT avec difficulté. «L'indice RTS des valeurs boursières cotées en Russie a gagné environ 3\% et dépassé la barre des 600 points. Une importante barrière psychologique franchie non sans peine. »

(21) Теннис. Андрееву не далась планка 1/8 финала Australian Open. Tennis. Andreevu ne da-l-as' planka.. Andreev-DAT NEG dat'-PASS-FEM-sja barre... «Tennis. Andréiev n'a pas passé la barre du 8ème de finale de l’Open d'Australie. »

Le sujet peut également être inscrit dans une téléonomie par dat'sja lui-même; on trouve alors des termes très variés : «disque », "popularité », "croissance », « choix », « combat », « instrument », « déménagement », « concert »... :

(22) По словам Дэвида Брауна этот диск дался ему тяжелее остальных. Po slovam Dèvida Brauna

ètot disk da-l-sja emu tjazhel-ee ostal'nyx.

ce disque dat'-PASS-SJA lui.DAT difficile-COMP autre-GEN.PLUR

«David Brown déclare que ce disque a été plus difficile à réaliser que les autres.» 
(23) Местный язык также поразительно легко дался ему, и сейчас он мог довольно бегло изъясняться на нем.

Mestnyjjazyk takže porazitel'no legko dalsja emu, Langue locale aussi étonnamment facilement dat'PASS-sja lui.DAT i sejčas on mog dovol'no beglo iz"jasnjat'sja na nëm.

«Il avait également assimilé la langue du pays avec une facilité déconcertante, et maintenant, il pouvait s'exprimer dans cette langue sans trop de peine. »

Dans tous ces exemples, le sujet renvoie non à une entité, mais bien à la représentation d'un procès impliquant cette entité, à savoir le procès $\mathbf{P}$ : barrière à surmonter, disque à réaliser, langue à apprendre etc. Quant au composant au datif, il s'agit d'un terme qui ne peut pas se constituer comme le constituant $\mathbf{D}$ apporté par dat'. Comparons cette construction avec celle, très ressemblante, illustrée par (24) :

(24) Ему встретился Иван.
Еmu vstreti-l-sja Ivan
Lui.DAT rencontrer-PASS-sja Ivan
«Il a rencontré Ivan (par hasard). » (litt. «À lui s'est rencontré Ivan »)

On entrevoit en quoi réside la différence entre les structures sans dat' et avec dat': dans ces dernières, qu'un terme agentif ne puisse pas se manifester en fonction sujet (suivant notre hypothèse de travail sur -sja dans ce cas) le prive également du statut de facteur $\mathbf{D}$ déterminant la validation du procès. Celle-ci est dès lors non effective, ou en bute à la force des choses. Dans (24), en revanche, que l'agent ne soit pas sujet ne s'oppose pas à la validation du procès : la rencontre, pour être fortuite, n'en a pas moins eu lieu.

\section{- Deuxième type d'emplois : exclamation}

Un emploi de dat'sja est glosé ainsi par le Dictionnaire de l'Académie en 17 volumes : «devenir l'objet de discussions sans fin, monopoliser l'attention ». La syntaxe en est le plus souvent réduite à sujet + dat'sja au passé + complément au datif :

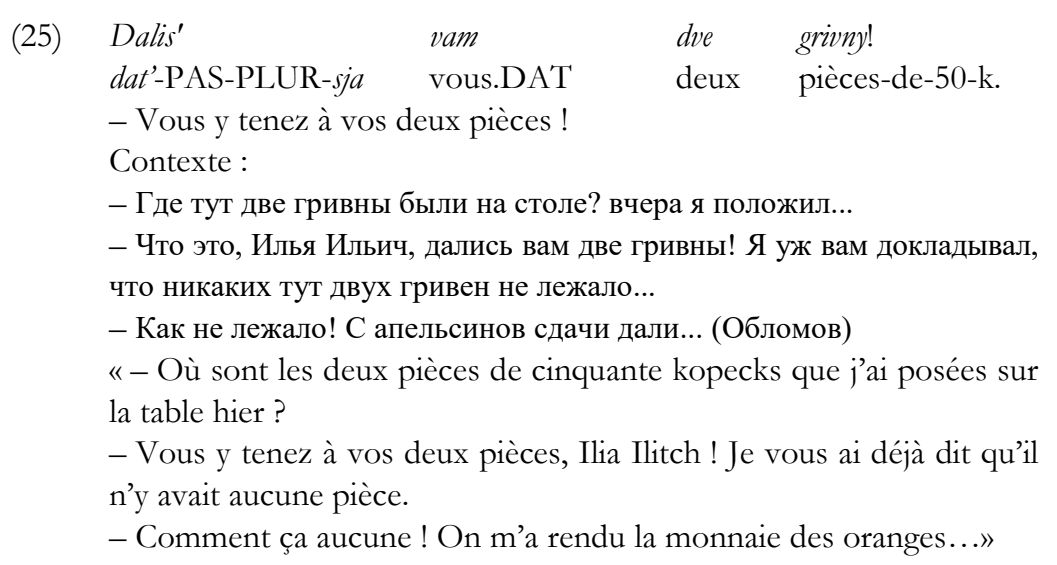


Le terme dve grivny « deux pièces » est présenté comme suscitant chez le sujet exprimé par le composant datif ("vous») un intérêt opiniâtre (cf. la traduction par "vos deux pièces ») et disproportionné. Le postfixe -sja entérine une absence : celle d'un référent venant instancier $\mathbf{D}$ pour autoriser la mise en relation « vous» / «les deux pièces ». Il s'ensuit que cette relation, qui correspond au terme $\mathbf{P}$ requis par le fonctionnement de dat', peut être mise en question en tant qu'objectif; ces énoncés comportent parfois les interrogatifs za $a \breve{G} m$ ou ăgo «à quoi bon»; en voici quelques exemples non commentés :

(26) Чего им эта Москва далась (...)

Cego im èta Moskva da-l-a-s'

À quoi bon il -DAT.PLUR cette Moscou dat'PASS-FEM-sja

"Qu'en ont-ils après Moscou? »

Contexte :

Международный порт под боком, таможня своя - чего им эта Москва далась, когда можно напрямую везти?

Mě̃dunarodnyj port pod bokom, tamožnja svoja - čego im èta Moskva dalas', kogda možno naprjamuju vezti?

«Un port international à disposition, une douane toute acquise qu'en ont-ils après Moscou, alors qu'ils peuvent faire la route directement?»

(27) Зачем она далась тебе?

Začem ona da-l-a-s' tebe

À quoi bon elle dat'-PASS.FEM toi-DAT

« Pourquoi t'obsède-t-elle à ce point? »

Contexte :

- Пусти меня, отец, туда.

- Куда?

- На ту звезду, что носит имя человека.

- О горе, горе мне. Эх ты, моя надежда, зачем она далась тебе? Ведь возвратившихся оттуда нету...

- Papa, laisse-moi y aller.

- Où ça ?

- Sur cette planète qui porte le nom d'un humain.

- Oh malheur! Voyons, espoir de ma vie, pourquoi t'obsède-t-elle à ce point? Tu sais bien que personne n'en est revenu...

\section{6. udat'sja « être une réussite »}

Au verbe non-pronominal dat' répond le substantif dača qui peut se traduire littéralement «octroi » : il désigne tantôt le procès de «cession ", tantôt un bien cédé, typiquement un bien immobilier, d'où le sens de «maison de campagne (jadis octroyée en récompense de services); datcha ». Dans ces deux interprétations sont impliqués deux sujets : un bénéficiaire ou nouveau propriétaire suivant que $\mathbf{P}$ représente une acquisition ou un état; et $\mathbf{D}$, condition sine qua non de $\mathbf{P}$. 
Il n'en va pas de même pour le dérivé en $u$ - : udača « réussite » implique bien un bénéficiaire (celui à qui profite la réussite), mais ce bénéficiaire n'est pas redevable à un tiers. On retrouve le mécanisme du passif modal, cette fois-ci en interaction avec $u$ Comparons le verbe udat'sja à dat'sja modal : dat'sja ne s'emploie pas sans complément adverbial, mais il n'en va pas de même avec udat'sja. Comparer :

(28) * Торт (мне) дался.

$\begin{array}{lll}\text { Tort } & (m n-e) & \text { da-l-sja } \\ \text { gâteau } & \text { (moi-DAT) } & \text { dat'-PASS-sja }\end{array}$

(29) Торт Аался мне кровью, потом и нервами.

$\begin{array}{llll}\begin{array}{l}\text { Tort da-l-sja } \\ \text { i nerv-ami }\end{array} & \text { mne } & \text { krov'-ju, } & \text { pot-om } \\ \text { gâteau dat'-PASS-sja } & \text { moi.DAT } & \text { sang-INSTR, } & \text { sueur-INSTR } \\ \text { et nerfs-INSTR.PLUR } & & \\ \text { "Le gâteau m'a coûté sang, sueur et nerfs. » } & \end{array}$

versus :

(30) Торт (мне) удался (на славу).

$\begin{array}{lllll}\text { Tort } & (m n-e) & u \text {-da-l-sja } & \text { (na } & \text { slav-u) } \\ \text { gâteau } & \text { (je-DAT) } & \text { u-dat'-PASS-sja } & \text { (à } & \text { perfection-ACC) } \\ \text { "Le (/mon) gâteau est une réussite (totale). » }\end{array}$

ou encore :

(31) * Инструмент дался.

$$
\begin{array}{ll}
\text { Instrument } & d a-l-s j a \\
\text { Instrument } & d a t \text {-PASS-sja }
\end{array}
$$

(32) Инструмент дался ему легко.

$\begin{array}{llll}\text { Instrument } & \text { da-l-sja } & \text { emu } & \text { legko } \\ \text { Instrument } & \text { dat'-PASS-sja } & \text { lui.DAT } & \text { facilement }\end{array}$

«Il maîtrisa vite l'instrument»

Contexte :

Инструмент дался ему легко, и вскоре Джо сам начал давать уроки игры.

Instrument da-l-sja emu legko, i vskore Dz̧ho sam načal davat' uroki igry « Il maîtrisa vite l'instrument, et bientôt Jo commença a donner luimême des leçons de guitare. »

versus :

(33) (au sujet de la première réalisation d'un luthier célèbre)

Инструмент удался.

Instrument uda-l-sja.
Instrument u-dat'-PASS-sja.
"L'instrument était réussi »

Le complément adverbial de (29) et (32) justifie l'impossibilité pour le sujet de figurer en qualité de $\mathbf{D}$ déterminant le procès sans partage: s'interposent des difficultés qu'il faut surmonter. Ce complément n'est pas nécessaire dans le cas de udat'sja $(30,33)$ parce qu'avec le préverbe $u$-, conformément à l'hypothèse reprise ici, le 
résultat du procès est indépendant des efforts investis par son agent et des écueils rencontrés.

\section{Conclusion}

Avec udat'sja, la notion de "réussite » est construite par combinaison des mécanismes respectifs à l'œuvre dans dat'sja et dans $u$. Qualifier une tarte de réussite au moyen du verbe udalsja à valeur de parfait, ou encore du substantif udača ${ }^{12}$, cela signifie d'une part que le procès $\mathbf{P}$ qui impliquant la tarte, à savoir la confection de la tarte, se trouve privé du facteur $\mathbf{D}$ déterminant sa validation (par conséquent, la réussite ne saurait être redevable à celui qui confectionne la tarte); de son côté, u caractérise l'état actuel de la tarte comme un développement inattendu du procès en question : tout repose in fine sur le jugement qui vient constater la réussite.

En somme, cette réussite-là échappe au pâtissier. Il en va de même pour un article : son auteur peut tout juste espérer avoir réussi, ou craindre l'inverse. 


\section{Travaux cités}

CAMUS R., 1998, «Quelques considérations sur le préverbe po- en russe contemporain », Revue d'Etudes Slaves, 70/1, 1998, p. 101-112. (cette publication et les suivantes sont en ligne sur la page internet de l'auteur).

CAmus R., 2007, «Leksema DAT': o leksičeskix posledstvijax grammatičeskix svojstv " «Le lexème russe DAT" : des conséquences lexicales des propriétés grammaticales », Materialy XXXVI meždunarodnoj filologičeskoj konferencii. 12-17 marta 2007 g. Sankt-Peterburg. Vyp. 14. Leksikologija i leksikografija (russko-slavjanskij cikl). - SPbGU, 2007.

CAMUS R., 2008 « Du slavon en russe contemporain : caractérisation générale et l'exemple de da à valeur optative ", in Construire le temps. Etudes offertes à J.P. Sémon, Paris, IES, 2008, p. $403-427$.

DE VogÜÉ, S., 1991, «La transitivité comme question théorique : querelle entre la théorie des Positions de J.C. Milner et la Théorie des Opérations Prédicatives et Enonciatives d'A. Culioli », in $\operatorname{LINX} \mathrm{n}^{\circ} 24$, p. 37-64

DObruŠInA E. R., MELLINA E. A., PAJJAR [=PAILlaRD] D., 2001, Russkie pristavki : mnogoznačnost' i semantičeskoe edinstvo (sbornik), M., Russkie slovari.

GORELIK E.V., 2001, «Opisanie glagol'noj pristavki u-», Moskovskij lingvističeskij そ̌urnal (tematičeskij vypusk: "Glagol'nye prefiksy i prefiksal'nye glagoly") 5/1, p. 37-68.

PAILLARD D., 2004, « À propos des verbes préfixés », Slovo 31, p. 13-44.

REY K, Ss date, Description du préverbe russe u- (usadit' etc.), Mémoire de maitrise soutenu sous la dir. de Chr. Bonnot, INALCO (mémoire déposé à la Bibliothèque de l'INALCO).

ROZINA R. I., 2005, Semantičskoe razvitie slova v russkom literaturnom jazylke i souremennom slenge, "Az̧bukounik », M.

SELIVËRSTOVA O. N., 1975, Komponentnyj analiz, mnogoznačnyx slov (na materiale nekotoryx russkix glagolov), M., Nauka.

SELIVËrstova O. N., 2004, Trudy po semantike, M. «Jazyki slavjanskoj kul’tury ».

ZALIZNJAK A. A., 2001, «Semantičeskaja derivacija v značenii russkoj pristavki 'u-', Moskovskij lingvističeskij žurnal, (tematičeskij vypusk: “Glagol'nye prefiksy i prefiksal'nye glagoly”) 5/1, p. 69-84.

ZASORINA, L. N., 1977, Castotnyj slovar' russkogo jasylka, M. 